\title{
Real-time 100 Gbps/ $\lambda /$ core NRZ and EDB IM/DD Transmission over 10 km Multicore Fiber
}

\author{
Rui Lin ${ }^{1,2}$, Xiaodan Pang, ${ }^{2,3}$, Joris Van Kerrebrouck ${ }^{3}$, Michiel Verplaetse ${ }^{3}$, Oskars Ozolins ${ }^{4}$, \\ Aleksejs Udalcovs ${ }^{4}$, Lu Zhang ${ }^{2}$, Lin Gan ${ }^{1}$, Ming Tang ${ }^{1}$, Songnian Fu ${ }^{1}$, Richard Schatz ${ }^{2}$, Urban Westergren ${ }^{2}$, \\ Sergei Popov ${ }^{2}$, Deming Liu ${ }^{1}$, Weijun Tong ${ }^{5}$, Timothy De Keulenaer ${ }^{6}$, Guy Torfs ${ }^{3}$, Johan Bauwelinck ${ }^{3}$, \\ Xin Yin ${ }^{3}$ and Jiajia Chen ${ }^{2}$ \\ (1) Huazhong University of Science and Technology, Wuhan, China, \\ (2) KTH Royal Institute of Technology, Electrum 229, Kista, Sweden, \\ (3) Department of Information Technology (INTEC) - IDLab, University of Ghent-imec, Belgium \\ (4) Networking and Transmission Laboratory, RISE Acreo AB, Kista, Sweden \\ (5) Yangtze Optical fiber and Cable Joint Stock Limited Company (YOFC), Wuhan, China \\ (6) BiFAST, spin-off of IDLab, Ghent University-imec, Ghent, Belgium \\ jiajiac@kth.se; tangming@hust.edu.cn
}

\begin{abstract}
A BiCMOS chip-based real-time IM/DD spatial division multiplexing system is experimentally demonstrated. $100 \mathrm{Gbps} / \lambda /$ core NRZ and EDB transmission is achieved below 7\%overhead HD-FEC limit after $10 \mathrm{~km}$ 7-core fiber with optical dispersion compensation. OCIS codes: (060.0060) Fiber optics and optical communications; (170.4090) Modulation techniques
\end{abstract}

\section{Introduction}

Emerging mobile and cloud applications drive ever-increasing capacity demands, particularly for short-to-medium reach optical communications, where intensity modulation and direct detection (IM/DD) system are preferable thanks to its low complexity. To address the growth of capacity demands, the research efforts should be put not only on scaling up the lane count but also increasing the single lane rate, e.g., to $100 \mathrm{Gbps}$ and beyond. In addition, realtime implementation is highly required for low latency services, and therefore heavy digital signal processing (DSP) needs to be avoided. Recent demonstrations over $100 \mathrm{Gbps}$ IM/DD systems were reported using advanced modulation formats, e.g. four-level pulse amplitude modulation (PAM-4) [1] and discrete multitone (DMT) [2]. Nevertheless, advancements in low power consumption and low complexity of the transceiver IC make simple nonreturn-to-zero (NRZ) [3] and partial response modulation format, such as electrical duo-binary (EDB) [4], attractive for high-speed real-time communications, especially when communications are achievable with simple analog equalizations.

Meanwhile, spatial division multiplexing (SDM) techniques provide a promising way to scale up the lane count per fiber, while reducing the number of fiber connections and patch cords, and hence simplifying cabling complexity. Recently, a real-time multicore fiber (MCF)-based IM/DD system [5] was reported. However, the lane rate (i.e., the data rate per wavelength per core) is limited to $25 \mathrm{Gbps}$. In this paper, we for the first time experimentally demonstrate a real-time IM/DD transmission system with $100 \mathrm{Gbps} / \lambda /$ core $\mathrm{NRZ}$ and EDB modulations over a 7-core fiber, which significantly advances the state-of-the-art lane rate while verifying its potential to scale up the lane count. Bit error rate (BER) below the 7\%-overhead hard decision forward error correction code limit (7\%-OH HDFEC) is achieved for $10 \mathrm{~km}$ transmission with dispersion compensation. The BiCMOS based transmitter and receiver chipset, the MCF and the fan-in/fan-out (FI/FO) devices used in the experiments are all in-house developed.

\section{Experiment setup}

The experiment setup is illustrated in Fig.1. A Xilinx Virtex Ultrascale FPGA generates four electrical 27-1 pseudorandom bit streams (PRBS) at $25 \mathrm{Gbps}$, which are shifted with a delay of 0, 32, 64 and 96 bits and multiplexed into a 100 Gbps NRZ signal by the transmitter (TX) IC. A six-tap analog feedforward equalizer (FFE) at the TX side is used to compensate the frequency roll-off and dispersion induced inter-symbol-interference in the link. A $50 \mathrm{GHz}$ $\mathrm{RF}$ amplifier is used to drive a C-band $100 \mathrm{GHz}$ distributed feedback electro-absorption modulated laser (DFBEAM) [6]. The DFB-EAM driven by $117.0 \mathrm{~mA}$ and biased at $-1.85 \mathrm{~V}$, emits at $1548.7 \mathrm{~nm}$. The output signal is amplified by an erbium-doped fiber amplifier (EDFA) and then divided into 7 branches using a 1x8 splitter. The split signals are further de-correlated with different delays and launched into the 7 cores of the MCF via a low-loss and low crosstalk FI/FO device, which is fusion spliced to the MCF at one end, split and connected to seven individual single mode fiber (SMF) pigtails at the other end. 


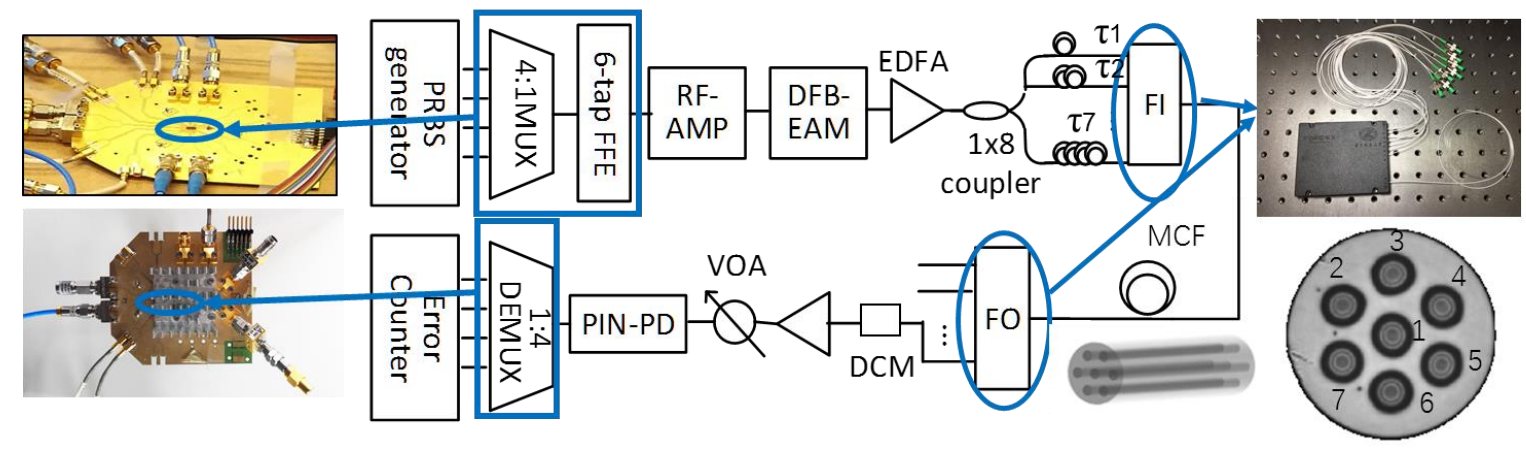

Fig. 1 Exeperiment setup.

After $10 \mathrm{~km} \mathrm{MCF}$ transmission, the spatial channels are demultiplexed by another FI/FO module so that the signals in each core can be connected to a SMF. A fixed dispersion compensation module (DCM) of $-159 \mathrm{ps} / \mathrm{nm}$ is used for coarse dispersion compensation. A variable optical attenuator (VOA) is employed to adjust the received optical power before the pre-amplifier and an InP-based PIN-PD (>90 GHz 3-dB BW) with a responsivity of $0.2 \mathrm{~A} / \mathrm{W}$. The received $100 \mathrm{Gbps}$ data is deserialized by the receiver (RX) IC into $4 \times 25$ Gbps streams for real-time error detection. The RX IC consists of 2 parallel level-shifting limiting amplifiers (LA), an XOR-gate and a 1-to-4 DEMUX. For EDB decoding, the threshold of both LAs need to be controlled to ensure the correct extracting of the upper and lower eye of the EDB signal from the varied received optical power. The NRZ detection, on the other hand, needs only one LA to be configured which can be kept constant ignoring the power fluctuation, which potentially reduces the complexity of the RX. The initial tap weights in the FFE for the NRZ or EDB are calculated by using a least square error algorithm to fit the linear combination of the impulse responses to an ideal pulse, as shown in Fig. 2(a) and (b). In the measurements, the tap weights are further adapted to minimize the BER. Both TX and RX ICs are designed in-house by the BiFAST team in a $0.13 \mu \mathrm{m}$ BiCMOS technology, occupies $1555 \mu \mathrm{m} \times 4567 \mu \mathrm{m}$ and $1926 \mu \mathrm{m} \times 2585 \mu \mathrm{m}$. The energy consumption of the TX and RX are less than $1 \mathrm{~W}$ and 1.2 $\mathrm{W}$, respectively [4].

The cladding diameter of the hexagonal MCF is $150 \mu \mathrm{m}$ and the core pitch is set as $42 \mu \mathrm{m}$. The crosstalk between adjacent cores is as low as $-45 \mathrm{~dB} / 100 \mathrm{~km}$. The average attenuation per core is less than $0.2 \mathrm{~dB} / \mathrm{km}$ at 1550 $\mathrm{nm}$. The cross-section view of the MCF is shown in Fig. 1. The FI/FO is realized by chemically etching the 7 bare SMF until the cladding diameter matches the MCF core pitch and arranging them in the hexagonal lattice [7].

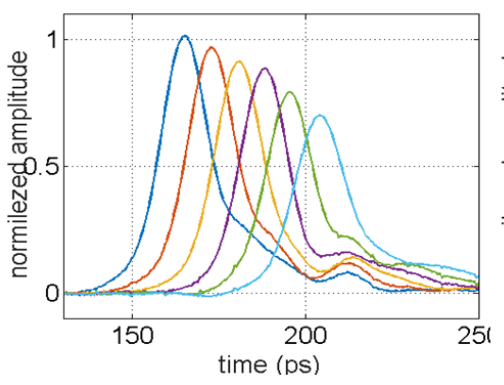

(a)

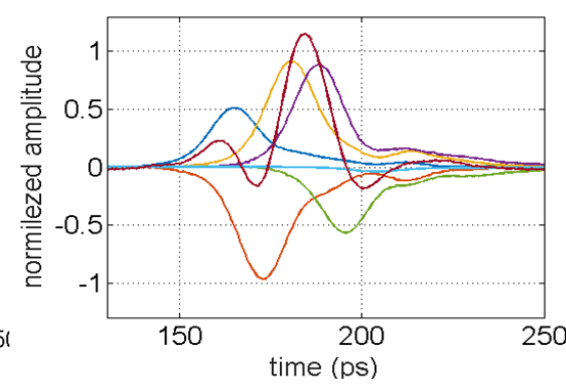

(b)

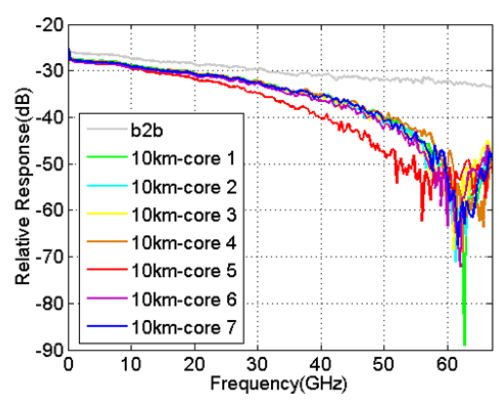

(c)

Fig. 2 (a) Impulse responses of each tap in the FFE, (b) linear combination with the optimum tap weights and (c) Frequency response of the b2b optical link and different cores of 10km MCF with DCM.

\section{Results and Discussions}

The frequency response of the optical back-to-back (b2b) and different cores of $10 \mathrm{~km} \mathrm{MCF}$ transmission with DCM is shown in Fig. 2(c). One can observe a frequency dip at around $62 \mathrm{GHz}$ across all the cores, indicating that a residual dispersion of $16 \mathrm{ps} / \mathrm{nm}$ is not fully compensated by the DCM. The variation among different cores is caused by insertion loss and end face reflection of the fabricated FI/FO devices. We evaluate the optical transmission performance of both NRZ and EDB at 100 Gbps using the experiment setup shown in Fig.1. The eye diagrams of the received NRZ and EDB signals in the optical b2b configuration are widely open (see Fig. 3(a) and (d), respectively) thanks to the broadband DFB-EAM and the PIN-PD. The end-to-end bandwidth of the link is mainly limited by the RX IC ( $41 \mathrm{GHz}$ ). Apart from the fixed DCM, the six-tap analog FFE at the TX is adjusted to enhance the system tolerance towards the imperfect dispersion compensation in different cores. 
The BER curves of the received optical signals after $10 \mathrm{~km}$ MCF transmission using NRZ and EDB are shown in Fig. 3(g) and (h), respectively. For the NRZ case, BER $=2.8 \mathrm{E}-6$ is reached and no error floor is observed in b2b configuration thanks to the broadband optical transceiver. $10 \mathrm{~km} \mathrm{MCF}$ transmission degrades the signal quality due to the chromatic distortion. The transmission in all the cores saturates at BER around 3E-3, below the 7\%-OH HDFEC. The power penalty compared to the $\mathrm{b} 2 \mathrm{~b}$ transmission ranges from $4.4 \mathrm{~dB}$ to $7.3 \mathrm{~dB}$ in different cores with an average value of $6.3 \mathrm{~dB}$. The eye-diagrams of the received signals after $10 \mathrm{~km}$ MCF transmission in core 4 and core 5 are shown in Fig. $3(\mathrm{~g})$, where the lowest and highest BER are observed at the received optical power of $\sim 8$ $\mathrm{dBm}$. Inter-symbol-interference due to the filtering effect of the fiber link is the main contributor to the eye closing.

In the EDB transmission, except for core 5, all the other spatial channels have similar receiver sensitivity to reach the $\mathrm{BER}=3.8 \mathrm{E}-3$ after $10 \mathrm{~km}$ MCF transmission. The sensitivity variation is mainly due to the end face reflection in the MCF link. EDB requires only half bandwidth compared to NRZ. As a result, less filtering effect over the link causes much lower penalty $(<2 \mathrm{~dB})$ compared to the $\mathrm{b} 2 \mathrm{~b}$ configuration. The eye diagrams of the EDB signals captured after $10 \mathrm{~km}$ in core 4 and core 5 are show in Fig. 3(e) and (f), respectively. A relatively better performance at the higher received optical power can be found in the EDB transmission, in which all the spatial channels are below the KP4 FEC limit (BER=2.0E-4).

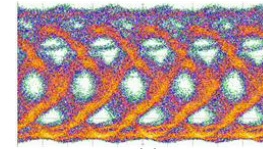

(a)

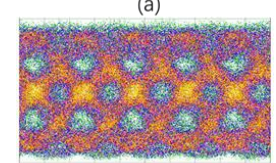

(b)

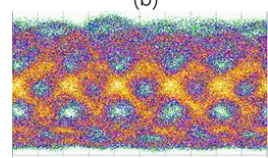

(c)

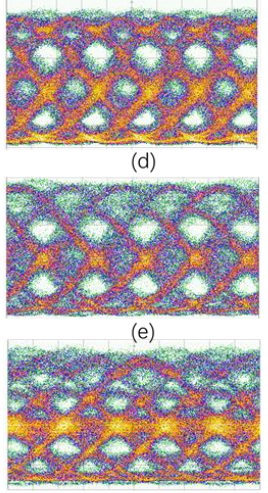

(f)

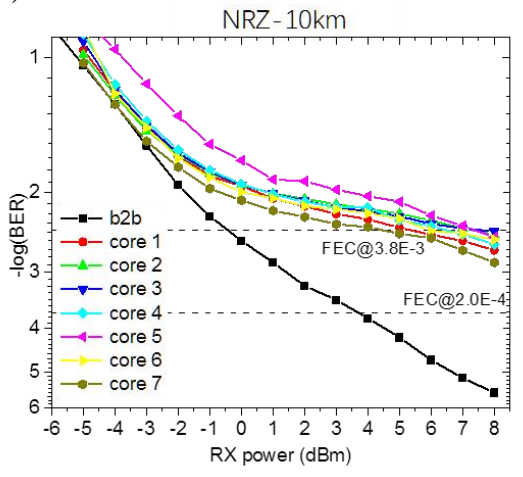

(g)

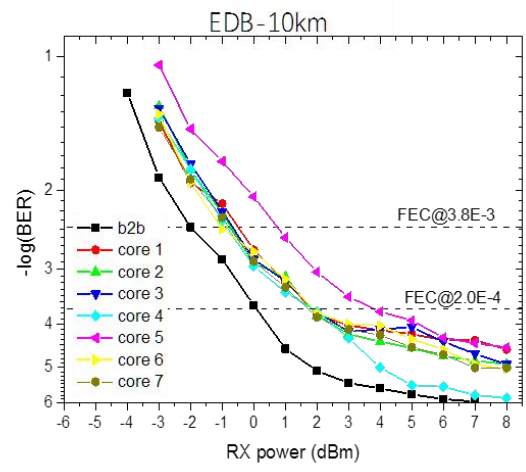

(h)

Fig. 3 Eye diagrams of the NRZ signals in (a) b2b, (b) core 4 and (c) core 5 after 10km MCF transmission, and the EDB signal in (d) b2b, (e) core 4 and (f) core 5 after 10km MCF; BER of the real-time 100Gbps (g) NRZ signals and (h) EDB signals measured for b2b transmission and different cores after $10 \mathrm{~km} \mathrm{MCF}$.

\section{Conclusions}

We experimentally demonstrated a MCF based real-time $100 \mathrm{Gbps} / \lambda /$ core IM/DD transmission system. BER below the 7\%-OH HD-FEC limit has been successfully achieved in the DSP-free dispersion compensated $10 \mathrm{~km} \mathrm{MCF}$ transmission for both NRZ and EDB signals.

\section{Acknowledgements}

This work was supported by Swedish Research Council (VR); the Swedish Foundation for Strategic Research (SSF); Göran Gustafsson Foundation, Swedish ICT-TNG, Flemish FWO \& IWT, IOF, Natural Science Foundation of Guangdong Province and National Natural Science Foundation of China.

\section{References}

[1] J. Lee, et al., "Demonstration of 112-Gbit/s optical transmission using 56GBaud PAM-4 driver and clock-and-data recovery ICs," in Proc. ECOC, PDP, 2015.

[2] Socionext Inc., "World's lowest-power IC for single-wavelength, 100Gbps transmission over SMF optical links," 2016.

[3] J. Verbist, et al., "Real-Time 100 Gb / s NRZ-OOK Transmission with a Silicon Photonics GeSi Electro-Absorption Modulator," in Proc. of OFC 2017.

[4] X. Yin, et al., "First demonstration of real-time 100 Gbit/s 3-Level duobinary transmission for optical interconnects," in Proc. of ECOC, PDP, 2016.

[5] Z. Feng, et al, "C-band real-time 400/300 Gb/s OOK bidirectional interconnection over 20 km multicore fibers," Chin. Opt. Lett., 15 (8), 2017.

[6] O. Ozolins, et al., "100 GHz EML for High Speed Optical Interconnect Applications,” in Proc. of ECOC 2016.

[7] B. Li, et al., "Role of wavelength dependent sensitivity in affecting the crosstalk mitigation of homogeneous multicore fiber: an analytical estimation approach," Opt. Express 22, 14127-14134, 2014. 